\title{
IAMJ
}

INTERNATIONAL

AYURVEDIC

MEDICAL JOURNAL

ISSN: 2320-5091

Impact Factor: 6.719

Review Article

\section{AN INTRODUCTION ABOUT SODHALA NIGHANTU}

\author{
Nishi Choudhary $^{1}$, S.K. Sharma ${ }^{2}$, Deepak Verma ${ }^{3}$, Priyanka ${ }^{4}$ \\ ${ }^{1,4}$ P.G. Scholar, ${ }^{2}$ Prof. and H.O.D., ${ }^{3}$ Assistant Professor \\ PG Department of Dravyaguna Vigyan, Dayanand Ayurvedic College, Jalandhar, Punjab, India
}

Corresponding Author: dr.nishi.choudhary@gmail.com

https://doi.org/10.46607/iamj1509022021

(Published online: February 2021)

Open Access

(C) International Ayurvedic Medical Journal, India 2021

Article Received: 15/01/2021 - Peer Reviewed: 28/01/2021 - Accepted for Publication: 07/02/2021

Check for updates

\section{ABSTRACT}

Ayurveda classics have many effective herbs to be evaluated for its own identity. In order to have a perfect understanding of the medicinal plants it is therefore most essential to be familiar with the branch of Ayurvedic Pharmacology i.e. "Dravyaguna Vigyan" which provides the scientific knowledge of the fundamentals of Ayurvedic medicinal properties with their pharmacological action. Ayurvedic history can be divided into three time periods Vedic period, Samhita period, Nighantu period. The Nighantu period is the golden period of the Ayurvedic Materia Medica literature. Many Nighantus or lexicons were written after the samhita period. Initially the Ayurvedic Nighantus are based on different samhitas containing only the synonyms which communicated the prevalent names, morphological characters, and properties, actions of drugs, habitat and relevant information. As time passed many species enters in India from their native places for economical and medicinal importance. Many of them were added in various lexicons on the basis of their pharmacological or therapeutic properties. Mainly the literature of Dravyaguna is available in two forms one is texts which describe only synonyms of drugs e.g. Ashtanga Nighantu, Paryaya Ratnamala, Hridaya Deepaka Nighantu, Nighantu Sesha, etc., and second is texts which explain synonyms along with properties, Indications and actions e.g. Dhanwantari Nighantu, Sodhala Nighantu, Madanpal Nighantu etc. Sodhala Nighantu is written by Acharya Sodhala in $12^{\text {th }}$ century. In the introductory verses Sodhala express his view that students of Ayurveda in his time are facing great difficulty as the list of drugs, proper classification, nomenclature etc. are not available. Therefore, he composed this work after con- 
sulting earlier works of Agnivesa, Sushruta and mainly the author of Ashtang - Vagabhattacharya. He claims that this text will solve controversies among various texts.

Keywords: Sodhala Nighantu, Ayurveda, Samhita, Medicinal Plants.

\section{INTRODUCTION}

Sodhala Nighantu is written by Sodhala in $12^{\text {th }}$ century. Sodhala is of brahmana caste born in vatsa gotra, belonging to traditional family of rayakavala. The rayakavala (rayekwad brahmins of vatsa gotra) vamsa belongs to Gujarat. Josi title is added or suffixed with his name. His father's name is Vaidya Nandana and he is disciple of Sanghadayalu. He is devotee of bhanu (surya) and he introduced himself as Jyotisha sasastri. He is referred in a Tamrapatra (copper plate inscription) belonging to Bhimadeva second. The book begins with the salutation before Lord Ganesha and text opens with offering prayer (Suryaradhana) to Lord Sun. The physician who is expert of medical science should gain erudition in the knowledge of nomenclature of drugs; hence, present contribution is made by compiling all the names of drugs as quintessence of drugs terminology. ${ }^{1}$

\section{Significance and Contribution}

There is major division (khanda) of whole work into two sections or parts (actually as two manuscripts dealing both subjects or aspects separately. This style of describing the drugs adopted by Sodhala is most practical and helping for understanding both aspects of drugs separately, for highlighting taxonomical as well as pharmacological importance of drugs individually. Further, the author has added new groups (Vargas) like Anekartha and Misraka varga in Namasangraha and $8^{\text {th }}$ varga to $27^{\text {th }}$ varga in Gunasangraha. It appears that Sodhala makes successful attempt for laying unique foundation in the field of Nighantu, by relieving the pharmacological aspects from nomenclatural material containing synonymy of drug and likewise, taxonomic aspect is made free from the facet of medicinal properties of drugs. Besides the Sanskrit terminology of drugs commonly forming the content of nighantu treatises in general, Sodhala has specifically concentrated on recording of regional terminology in order to make the description of drug more un- derstandable, easy and practicable. This style of Sodhala appears to be quite natural, applied and simple, so that any layman can recognize a source plant growing and available for particular drug (ausadha dra$v y a$ ), on the other hand, the physician will take help to identify, verify and determine the identity of drug and its plant source, which is generally known by specific local name. There are various specialties which are found remarkable in this treatise such as the determination of properties of drugs and dietary articles (Ausadha Khadyaahara) by their taste in regard to unaccounted items (or Dravyas), affinity of medicinal properties of products (like gum or latex) with source plant, production of complications caused by Guna of meals (Ahara) in excess, mention of Tanucchada in various points of hygiene (Svasthavrtta). Sodhala incorporates drugs from all the sources i.e. botanical, geological and zoological, with predominance of the drugs from vegetable kingdom. The numerical survey of dravyas (Drugs and allied substances) finds near about 500 in number in the first part named as $\mathrm{Na}$ masangraha (comprising mainly 7 vargas), while this number goes high if the dravyas (e.g. dhanyas - cereals, paddy etc. and other liquids etc.) are accounted for (other vargas: 1-22 and further dietics and other, in vargas exceeding to 27 vide gunasangraha, second part of work). Among the classified groups (Vargas), various groups of drugs (Ausadha Vargas) are named similar to other works of Nighantu except for Laksmanadi varga (no. 7 in both the parts). Thus, the creation of Laksmanadi varga appears to be new contribution of Sodhala Nighantu, especially for its composition of various drugs of uncommonly. Nearabout 68 drugs (mostly plants drugs except 1 or 2 probably) are incorporated. ${ }^{2}$ 


\section{DISCUSSION}

PART 1-The first part of the book have 7 chapters containing names and last two chapters i.e. $8^{\text {th }}$ and $9^{\text {th }}$ anekarthvarga as well as misrakadhyaya. Thus, there are 9 chapters in Nama Sangraha.

\section{PRATHAMA - GUDUCHYADI VARGA}

\section{The drugs are as follows:}

Guduchi (Tinospora cordifolia (Willd) Miers) Ativisha (Aconitum heterophyllum Wall), Murva (Marsedenia tenacissima W\&A), Morata (Maerua arenaria Hook )- It is a variety of Murva, Manjistha (Rubia cordifolia Linn), Dhanvayasa (Fagonia Arabica Linn), Yasa (Alhagi pseudalhagi Desv)-It is a variety of Dhanvayasa, Vasaka (Adhatoda vasica Nees), Khadira (Acacia catechu Willd), Somavalka (Acasia suma Kurz)- It is a variety of Khadira. Nimba (Azadirachta indica A. Juss), Mahanimba (Melia azadarach Linn), Kiratatikta (Swertia chirata Buchham), Katuka (Picrorhiza kurroa Royle ex Benth), Mustha (Cyperus rotundus Linn, Parpata (Fumaria indica Pugsley), Hribera (Coleus vettiveroides K. C. Jacob), Patola (Trichosanthes dioica Roxb), Swadu patra phala- It is Patola variety. Haridra (Curcuma longa Linn), Daru haridra (Berberis aristata DC), Shati (Hedychium spicatum Buch Ham), Gandhapalashi- It is a variety of Shati. Pushakara moola (Inula racemoa Hook), Bharangi (Clerodendrum serratum Spreng), Patha (Cissampelos pareira Linn), Shwasari- It is variety of Patha. Katphala (Myrica nagi Thunb), Devadaru (Cedrus deodara Linn), Katruna (Cymbopogon martini Roxb), Kapatam- It is Katruna variety. Guntha (Typha elephantine Roxb), Shrungi (Pistacia integerrima Stew. ex. Brandis), AjashrungiVariety of Shrungi. Shalaparni (Desmodium gangeticum DC), Shalaparni vishesha, Prushniparni (Ureria picta Desv), Prishnaparni vishesha, Bruhati (Solanum indicum Linn), Kantakari (Solanum xanthocarpum Schrad and Wendl), Lakshmana, Kasagni, Vruntaki, are the varieties of Bruhati. Gokshura (Tribulus terrestris Linn), Bilva (Aegle marmelos Corr), Agnimantha (Premna latifolia Roxb, Clerodendrum phlomidis Linn), Shyonaka (Oroxylum indicum Vent), Kashmarya (Gmelina arborea Linn), Patala (Stereospermum suaveolens DC), Kasthapatala- It is Patala vish- esha. Jeevaka (Microstylis wallichi Lindl), Rishabhaka (Microstylis muscifera Ridley), Meda-Mahameda (Polydonatum Verticillatum All), Vijaya- It is Meda vishesha. Also called Bhang (Cannabis sativa Linn), Kakoli (Roscoea procera), Ksheerakakoli- It is one of the Roscoea species. Mashaparni (Teramnus labialis Spreng), Mudgaparni (Phaseolus trilobus), Jeevanti (Leptadenia reticulata W\&A), Madhuyasthi (Glycyrrhiza glabra Linn), Kleetanakam - A variety of Yasthimadhu, Riddhi (Habenaria sp), Vidarika (Pueraria tuberosa DC), Ksheeravidari (Ipomoea digitata Linn), Kapikacchu (Mucuna pruriens DC), Dadhipushpi- It is a Kapikacchu vishesha. Sheetavara (Celosia argentea Linn), Pashanabheda (Bergenia ligulata Wall), Shravani-Mahasharavani (Sphaeranthus indicus Linn, Sphaeranthus africans Linn), Sariva (Hemidesmus indicus R. Br), Krishna Sariva (Cryptolepis buchanani Roem.\& Schult.), Bakuchi (Psoralea corylifolia Linn), Madana (Randia dumetorum Lam), Iksvaku (Lagenaria vulgaris Ser), Jeemutaka (Luffa achinata Roxb), Trapusa (Ccumis sativus Linn), Urvaru (Cucumis utilissimus Roxb), Valukam, Sheerna vrintam, Dangari, Vandhya karkotaki, Karkotaki (Mimordica dioica Roxb) are Trapusa vishesha. Chirbhita, Kushmandika (Benincasa cerifera Savi), Dhamargava (Luffa aegyptiaca Mill ex Hook), Koshataki (Luffa acutangula Linn, Ashmantaka (Ficus cordifolia Roxb), Kovidara (Bauhinia purpureaLinn), Visanika (Gymnema sylvestre R. Br.) ,Shanapushpi (Crotalaria verrucosa Linn), Bimbi (Coccinia indica W.\&A.), Haritaki (Terminalia chebula Retz), Vibhitak (Terminalia belerica Roxb), Amalaki (Phyllanthus emblica Linn), Aragvadha (Cassia fistula Linn), Karnikara- An Aragvadha vishesha. Danti (Baliospermum montanum Muell), Varani- A Danti vishesha. Jyapala (Croton tiglium Linn), Dravanti, Nilini (Indigofera tinctoria Linn), Snuhi (Euphorbia nerifolia Linn), Satala, Ksheerini-Swarna ksheeri (Euphorbia thomsoniana Boiss, Argemone Mexicana Linn), Shyama (Operculina terpethum Silva manso), Shuklabhandi- A Shyama vishesha, Indravaruni (Citrullus colocynthis Schrad), Vishala (Trichosanthus palmate Roxb), Shetapushpi- It is an Indravaruni vishesha. Trayamana (Gentian kurroo Royle), Ya- 
vatikta, Ankota (Alangium salvifolium Linn), Apamarga (Achyranthes aspera Linn), Raktapushpa- an Apamarga vishesha, Tejaswini, Jyotishmati (Celastrus paniculataus Willd), Rasna (Pluchea lanceolata Oliver \& Hiern), Ashwagandha (Withania somnifera Dunal), Punarnava (Trianthema portulacastrum Linn), Rakta Punarnava (Boerhavia diffusa Linn), Saireyaka- has three species -Sheta rakta (Barleria cristata Linn), Peeta (Barleria prionitis Linn), Neela (Barleria stribosa Linn). Bala (Sida cordifolia Linn), Mahabala (Sida rhombifolia Linn), Naagabala (Grewia hirsuta Venb.), Atibala (Abutilon indicum Linn), Prasarini (Paederia foetida Linn), Shatavari (Asperagus racemosus Willd), Sahasra virya (Asperagus sarmentosus Linn), Eranda (Ricinus communis Linn). ${ }^{3}$

\section{Dwitiya - Shatapushpadi Varga}

Shatapushpa (Peucedanum graveolens Linn), Mishreya (Foeniculum vulgare Mill), Vacha (Acorus calamus Linn), Hapusha (Juniperus communis Linn), Vidanga (Embelia ribes Burm), Kutaja (Holarrheana antidysenterica Wall), Indrayava, Yavakshara, Swarjikshara, Tankana kshara, Saindhava, Vida lavana, Sauvarchala, Audhbhida lavana, Samudra lavana, Hingu (Ferula foetida Regel), Hingu patri, Nadi hingu (Gardenia gummifera Linn), Tumburu (Zanthoxylum alatum Roxb), Sukshma ela (Elettaria cardamomum Maton), Bhadra ela (Amomum subulatum Roxb), Nagakeshara (Mesua ferrea Linn), Twak (Cinnamomum cassia Blume), Tamala patra (Cinnamomum tamala Nees \& Eberm). Talisa patra (Abies webbiana Lindl), Vamsha lochana (Bambusa arundinaceae Willd.), Upakunchi (Nigella sativa Linn), Dadima (Punica granatum Linn), Dhanyaka (Coriandrum sativum Linn), Jeeraka, Shukla jeeraka (Cuminum cyminum Linn), Krishna jeeraka, Bruhatpali- Are Jeeraka vishesha, Pippali (Piper longum Linn), Pippali moola- Piper longum., Chavika (Piper chaba Hunter), Gajapippali - It is the fruit of Chavya. Chitraka (Plumbago zeylanica Linn), Shunthi (Zingiber officinale Roscoe), Ardraka, Maricha (Piper nigrum Linn), Shetamaricha- The fruit of Shigru beeja. Yavani (Trachuspermum ammi Linn), Chauhara (Artemisia maritime Linn), Yavani (Hyoscymus niger
Linn), Vrukshamla (Garcinia indica), Amlavetasa (Garcinia prdunculata Roxb), Ajamoda (Trachyspermum roxburghianum DC), Ajagandha, Kapittha (Feronia elephantum Correa), Sharakara, Madhu sharakara, Yavasa sharakara are Sharakara vishesha. ${ }^{4}$

Tritya - Chandanadi Varga

Chandana (Santalum album Linn), Rakta Chandan (Pterocarpus santalinus Linn), Kuchandana (Caesalpinia sappan Linn), Kaliyakam (Yellow variety of Chandan), Barbarikam- a variety of Chandan. Kumkuma (Crocus sativus Linn), Ushira (Vetiveria zizanioides Linn), Priyangu (Callicarpa macrophylla Vahl), Tooni (Cedrela toona Roxb), Gorochana (Bezoar), Turushka (Aitingia excelsa Noronha.), Agaru (Aquilaria agallocha Roxb), Kaleyaka- An Agaru vishesha. Kasturi (Moschus moschiferus Linn), Karpura (Cinnamomum camphora Nees \& Eberm), Jatipatri (Myristica fragrans Houtt)- The red outer covering of the Jatiphala is known as Jatipatri. Jatipahala (Myristica fragrans Houtt), Kankola (Piper cubeba Linn.f.), Puga (Areca catechu Linn), Lavanga (Syzygium aromaticum Linn), Nalika, Nalada (Nordostachys jatamansi DC.), Gandha mansi (Selinum tenuifolium Wall), Kustha (Saussurea lappa C.B. Clarke), Renuka (Piper aurantianum Wall.), Tagara (Valeriana wallichii DC.) Pariplavam- a Cyperus sp. Nakha (Helix aspera), Vyaghra nakha, Sprkka (Delphinium zalil Aitch \& Hemsl), Bola (Commiphora myrrha Holmes), Damana (Artemisia vulgaris Linn), Dama- Damana vishesha. Mura (Selinum sp), Sthounayeka (Taxus baccata Linn), Coraka (Angelica glauca Edgw), Saileya (Parmelia perlata Ach), Elavaluka (Prunus cerassus Linn.), Sarala (Pinus longifolia Roxb), Saptaparna (Alstonia scholaris R. Br), Laksha (Lac), Amalaki (Embelica officinalis), Lamajjaka, Padmaka (Prunus puddum Roxb. Ex Wall), Dhataki (Woodfordia fruticosa Kurz), Prapaundarika, Karchura (Curcuma zedoaria Rose.), Manashila (Realgar), Sindhura (Redlead), Girisindhura, Saurashtri mruttika (Multani mitti), Gandhaka (Sulphur), Vatasaugandhika, Ambasthaka (Tamarix articulata Vahl.), Siktha (Wax), Raala, Kasisa (Iron sulphate), Pushpa kasisa- a variety of kasisa, Guggulu (Commiphora mukul Hook.ex Stocks), Kunduru (Boswellia sarrata 
Roxb.) it is Sallakiniryasa, Shrivestaka (Pinus longifolia Roxb.), Shallaki (Boswellia serrata Roxb), Kampillaka (Mallotus philippinensis Muell Arg), Kankustha, Bhallataka (Semicarpus anacardium Linn), Tuttha (Copper sulphate), Artha (Shilajatu) Suvarna makshika (Copper pyrite), Tapi (vimalmakshika), Anjana (Galena), Samudraphena (Sepia officinalis), Chakshushya (Cassia absus Linn), Rasanjana, Pushpanjana (Zinc oxide), Gairika (Ochre), Suvarna gairika, Kataka (Strychnos potatorum Linn), Lodhra (Symplocos racemosa Roxb), Kramuka- A Lodhra vishesha. Shankha (Conch). ${ }^{5}$

\section{Chaturtha - Karaviradi Varga}

Karaveera (Nerium indicum Mill), Chakramarda (Cassia tora), Dhattura (Datura alba Linn), Langali (Gloriosa superba Linn),Mahaloti, Moti, Untakantalo( Echinops echinatus), Dramathiu kachur, Musal(Chlorophytum borivianum), Timir vraksha, Bhringaraja (Eclipta alba Linn), Arka (Calotropis procera R. Br), Rajarka (Calotropis gigantea Linn), Buka (Osmanthus fragrans Lour.), Kakamachi (Solanum nigrum Linn), Kakajangha (Peristrophe bicalyculata Nees.), Kakanasa (Martynia diandra Glox.), Kakadani, Mulaka (Raphanus sativus Linn), Chanakyamulaka, Grinjana- are variety of Moolaka, Shigru (Moringa pterygosperma Gaertn), Sarshapa (Brassica compestris Prain.), Rajakshavaka- a variety of sarshapa, Bhutika ( Cymbopogon schoenanthus Linn.), Surasa (Ocimum sanctum Linn), Jambhira (Cymbopogon citrates Staff.), Kutheraka, Shaluka, Sumukha (Ocimum basilicum Linn.), Asuri (Brassica juncea Linn), Gandira (Ranunculus scleratus Linn.), Gaja pippali, Rasona (Allium sativum Linn.), Mahakanda- A Rasona variety, Garjar (Daucus carota DC.), Palandu (Allium cepa Linn), Kshirapalandu, Kadali (Musa sapientum Linn), Kastha kadali- A Kadali vishesha, Sindhuvara (Vitex negundo Linn), Nirgundi (Vitex negundo Linn.) Girikarnika (Clitorea ternatea Linn.), Jantukari, Padma, Varahi (GrusthiDioscorea bulbifera Linn), Mamsarohini (Soymida febrifuga A. Juss), Vandaka (Loranthus longiflorus Desr), Adityakanta (Gynandropsis pentaphylla DC.), Bramhi- a Suvarchala vishesha, Nakuli (Rouwolfia serpentina Benth ex Kurz), Gandha nakuli (Ophior- rhiza mungos Linn.), Vriddhadaruka (Argyreria speciosa Sweet), Raktapadi (Mimosa pudica Linn), Shankhapushpi (Convolvulus pluricaulis Chois), Vishnukranta- A Shankhapushpi vishesha, Tanduliya (Amaranthus spinosus Linn), Kasamarda (Cassia occidentalis Linn), Ikshu (Saccharum officinarum Linn), Guda (Jaggery), Kasha (Saccharum spontaneum Linn), Munja, Darbha (Imperata cylindrical Beauv), Kusha (Desmostachya bipinnata Stapf), Shara (Saccharum officinarum Linn.), Vamsha (Bambusa arundinaceae Willd), Nala (Phragmites kirka Trin), Mahanala- A variety of Nala, Durva (Cynodon dactylon Linn), Shweta durva, Gandadurva, Shweta utpala ( Pundrika), Nilotpala (Nymphaea stellata), Rakta utpala, Kumuda and Shudrotpala- Are variety of Pundarika, Padmini (Nelumbo nucifera Gaertn), Padmabeeja, Mrunala, Padmamula, Padmakesara. ${ }^{6}$

\section{Panchama- Amradi Varga}

Amra (Mangifera indica Linn), Kshudramra (Schleichera trijuga Willd), Rajamra- An Amra vishesha, Amrataka (Spondias mangifera Willd), Jambira (Citrus limon Linn), Madhu jambeera- A Jambeera vishesha, Naranga (Citrus reticulata Linn), Bijapuraka (Citrus medica Linn), Amlika (Tamarindus indica Linn), Kshudramlika- An Amlika vishesha, Aruka (Prunus persica Batsch), Bhavya (Dillenia indica Linn), Tinduka (Diospyros embryopteris Pers), Vikankata (Flacourtia romantchii L.Herit), Madhuka (Basia latifolia Roxb), Jalaja- A Madhuka vishesha, Pilu (Salvadora persica Linn), Kharjuri (Phoenix sylvestris Roxb), Pinda Kharjura (Phoenix dactylifera Linn), Draksha (Vitis vinifera Linn), Uttarapathika- A Draksha vishesha, Akshoda (Juglans regia Linn), Parushaka (Grewia asiatica Linn), Tuda (Morus indica Griff), Palevata, Tala (Borassus flabelifer Linn), Maad-A Taala vishesha, Piyala (Buchanania latifolia Roxb), Narikela (Cocos nucifera Linn), Vata (Ficus bengalensis Linn), Ashwattha (Ficus religiosa Linn), Plaksha (Ficus infectoria Roxb), Jambu (Eugenia jambolana Lam), Udumbara (Ficus glomerata Roxb), Kakodumbarika (Ficus hispida Linn), Rajadana (Mimusops hexandra Roxb), Shleshmataka (Cordia myxa Roxb), Shami (Prosopis specigera Linn), Badara (Zizyphus sativa), Kola (Zizyphus jujuba Lam), Kar- 
kandhu (Kshudra Badara) (Zizyphus nummularia W\&A), Karira (Capparis aphylla Roth), Karamarda (Carissa carandus Linn), Kadamba (Anthocephalus cadamba Mig), Karanja (Pongamia pinnata Linn), Udakirya, Angara valli, - Are Karanja vishesha, Shirisha (Albizzia lebbeck Benth), Arjuna (Terminalia arjuna W \& A), Vetasa (Salix caprea Linn), Varuna (Crataeva nurvala Buch-Ham), Shinshipa (Dalbergia sissoo Roxb), Sarja (Vateria indica Linn), Ashwakarana (Dipterocarpus alatus Roxb), Shala (Shorea robusta Gaertn), Asana (Mahasarja) (Pterocarpus marsupium Roxb), Shalmali (Bombax malabaricum DC), Rohitaka (kutasalmali) (Tecomella undulata Seem), Mushkaka (Schrebera swietenioides Roxb), Irimeda (Acacia farnesiana Willd), Mallika (Jasminum sambac Ait), Varshiki (Jasminum sp), Jati (Jasminum grandiflorum Linn), Vasanti (Jasminum arborescens Roxb), Graishmi (Jsminum sp), Champaka (Michelia champaka Linn), Taruni (Rosa centifolia Linn), Yuthika (Jasminum auriculatum Vahl), Svarnayuthika and Sankhayuthika are types of yuthika. Kunda (Jasminum pubescens Willd), Amilata, Atimuktaka (Hiptage madablota Gaertn), Bakula (Mimusops elengi Lam), Kinkirata (Garuga pinnata Roxb), Tilaka (Wendlandia exerta DC), Ashoka (Saraca ashoka Linn), Kimshuka (Butea frondosa Koen ex Roxb). ${ }^{7}$

\section{Shashtha - Suvarnadi Varga}

Suvarn (Gold), Raupya (Silver), Tamra (Copper), Trapu (Tin), Reetika (Brass), Seesaka (Lead), Kansya (Bell metal), Loha (Iron), Varta loha, Lohocchistha A Loha vishesha, Parada (Mercury), Hingula- Parada choorna vishesha. ${ }^{8}$

\section{Saptma - Laksamanadi Varga}

Laksmana, Mayursikha (Actinopteris dichotoma Bedd.), Kitagandha, Alpakacchuka (Mucuna prurita Hook.), Nakanari, Girimadhya (Coleus barbatus Benth.), Syavidudaraja (Porcupine), Sallaka, Gudadilla, Nahi, Kumari (Aloe barbadensis Mill.), Kaccaka (Xanthium strumarium Linn.), Maitri, Dundubhati, Tuvaraka (Hydnocarpus wighti ana Blume.), Somaraji (Psoralea corylifolia Linn.), Durmila, Kuberaksi, Rohita, Grdhranakhi (Himsra), Parpoti (Physalis minima Linn.), Bhilla, Nimbi, Ksutkari (Centipedia orbicu- laris Lour.) Modayanti, Asradana, Markosa, Valliyaka, Naraci, Mayuka (Quercus infectoria Oliver.), Dugdhika, Jvalatpuspa, Kataha, Lingbheda, Asmavalkala, Bhringi (Hymenodictyon excelsum Wall.), Rajasami, Ghantavali (Crotalaria sp.), Kadamba (Anthocephalus cadamba Miq.), Taksaki, Kembuka (Costus speciosus Koen.), Madhindhu, Panasi, Siddhesvari (Delonix elata Gamb.), Gangati, Kanchani, Visakarprika (Trianthema portulacastrum Linn.), Kakandola, Sarapunkha (Tephrosia purpurea Linn.), Pasani, Avanti, Jayavanti, Ksharapinda, Uttandaka (Echinops echinalus Linn.), Adhahpuspi, Hamsapadi (Adiantum lunulatum Burm.), Bachrula, Bandhuka, Sringirittika (Pentatriopis microphyliia W\& A), Srinkhala, Ghotaka, Koranta, Jiravali (Leea sp.), Mayurakhya bija, Visatinduka (), Akarakarabha (Anacyclus pyrethrum DC.), Svetavatu, Sphurdana, Devgandhari, Madhyanika (Bandhuka) (Pentapetes phoenicea Linn.), Jingini, Palasini (Butea superba Roxb.), Kasthahva, Mahadevi, Bijaka (), Narapuspaka, Iksura (Hygrophila spinosa T. Adersen.), Darakrishna, Musali (Curculigo orchioides Gaertn.), Masali (Mamsadravana), Angaraketu (Mamsavilasaka), Jalajambu (Prachibala) (Eugenia heyneana Wall.), Maksika, Maharastri, Usandi (Missata), Ingudi(indian almond ), Munivrksa, Godhamati, Kambojipatra, Vrddhapalitaka. ${ }^{9}$

\section{Ashtma-Anekartha Varga}

In this varga, there are 5 divisions- group of drug-term with double meaning ends, group of drug-terms with triple meaning ends, group of drug-terms with tetra meaning ends, group of drug-terms with penta meaning ends. ${ }^{10}$

\section{Navama-Mishrakadhaya}

This chapter contains following:-

Saktu-Mantha, Panaka-Laja, Sugandhi yoga, Triphala, Svadu Triphala, Sugandhi Triphala, Trikatu, Lavanatraya, Ksara-ksara, Trisama-Samatrika, Madhura Trayam, Chaturbhadra, Trikarsika, Trijata, Chaturjata, Chaturthaka, Panchakola, Panchakolaka Dvitiyakam, Panchaksiri Vrksa, ${ }^{11}$ and

\section{Parisistam}


This chapter contains following: -

Jala, Dugdha, Dadhi, Navnita, Ghola, Ghrita, Madhu, Kanji. ${ }^{12}$

PART 2- Guna Sangrah can be divided into three groups.

Group $1^{\text {st }}:^{13}$

- Prelude

- Dravyavali (Index): Content of Drugs [Groups]

Group 2 ${ }^{\text {nd: }}{ }^{14}$

Rasa-Virya-Vipaka-Prabhava Karana gyananam

Group $3^{\text {rd }}{ }^{15}$

Explain the varieties, properties and therapeutic indications of the drugs explained in major groups of $\mathrm{Na}$ masangraha part.

\section{Aushadh Vargas (Group of Drugs):}

Guduchyadi Varga; Shatapushpadi Varga; Chandanadi Varga; Karviradi Varga; Amradi varga; Suvarnadi Varga; Lakshmanadi Varga

\section{Aahar Vigyanam (Dietetics Groups):}

Paniya Varga; Kshira Varga; Dadhi Varga; Takra Varga; Navaneet Varga; Ghrita Varga; Taila Varga; Madhu Varga; Ikshu Varga; Madya Varga; Mutra Varga; Mutra Varga; Shuka Dhanya Varga; Jurna Varga; Trinadhanya Varga; Shimbidhanya Varga; Kritannna Varga; Anupan Varga; Mamsa Varga; Mishrakadhyaya.

\section{CONCLUSION}

Among all the lexicons, Sodhala Nighantu is the first well defined Nighantu which divides synonyms (Namarupa Vigyan) and properties of drugs exclusively. This Nighantu gives complete knowledge of Dravyaguna Vigyan which is a famous branch of Ayurve$d a$. This can help in treatment of various diseases and also in research purpose about various concepts of Ayurveda. Sodhala Nighantu gives good description about dietetics which is an emerging branch of medical science nowadays. This Lexicon also described the properties of metals and minerals which are essential for human body.

\section{REFERENCES}

1. Prof. (Dr.) Gyanendra Panday,Acharya Sodhala's Sodhala Nighantu Text With English- Hindi Commen- taries; Chowkhamba Krishnadas Academy Varansi, Edition First 2009, Preface PgNo 13

2. Prof. (Dr.) Gyanendra Panday, Acharya Sodhala's Sodhala Nighantu Text With English- Hindi Commentaries; Chowkhamba Krishnadas Academy Varansi, Edition First 2009, Preface PgNo 23-28

3. Umakant N. Rabb. Review on Dhanvantari Nighantu An Important Ayurvedic Lexicon | August 2018 | Vol 6 | Issue 8, Issn: 2322 - 0902 (P) Issn: 2322 - 0910 (O), PgNo 62-68 / Prof. (Dr.) Gyanendra Panday, Acharya Sodhala's Sodhala Nighantu Text With English- Hindi Commentaries; Chowkhamba Krishnadas Academy Varansi, Edition First 2009 PgNo 22-55

4. Prof. (Dr.) Gyanendra Panday, Acharya Sodhala's Sodhala Nighantu Text With English- Hindi Commentaries; Chowkhamba Krishnadas Academy Varansi, Edition First 2009, PgNo 55-68

5. Prof. (Dr.) Gyanendra Panday,Acharya Sodhala's Sodhala Nighantu Text With English- Hindi Commentaries; Chowkhamba Krishnadas Academy Varansi, Edition First 2009, PgNo 68-90

6. Prof. (Dr.) Gyanendra Panday, Acharya Sodhala's Sodhala Nighantu Text With English- Hindi Commentaries; Chowkhamba Krishnadas Academy Varansi, Edition First 2009 PgNo 91-107

7. Prof. (Dr.) Gyanendra Panday, Acharya Sodhala's Sodhala Nighantu Text With English- Hindi Commentaries; Chowkhamba Krishnadas Academy Varansi, Edition First 2009 PgNo 107-124

8. Prof. (Dr.) Gyanendra Panday, Acharya Sodhala's Sodhala Nighantu Text With English- Hindi Commentaries; Chowkhamba Krishnadas Academy Varansi, Edition First 2009 PgNo 124-126

9. Prof. (Dr.) Gyanendra Panday, Acharya Sodhala's Sodhala Nighantu Text With English- Hindi Commentaries; Chowkhamba Krishnadas Academy Varansi, Edition First 2009 PgNo 126-138

10. Prof. (Dr.) Gyanendra Panday, Acharya Sodhala's Sodhala Nighantu Text With English- Hindi Commentaries; Chowkhamba Krishnadas Academy Varansi , Edition First 2009 PgNo 138-149

11. Prof. (Dr.) Gyanendra Panday, Acharya Sodhala's Sodhala Nighantu Text With English- Hindi Commentaries; Chowkhamba Krishnadas Academy Varansi, Edition First 2009 PgNo 150-156

12. Prof. (Dr.) Gyanendra Panday, Acharya Sodhala's Sodhala Nighantu Text With English- Hindi Commentaries; Chowkhamba Krishnadas Academy Varansi, Edition First 2009 PgNo 156-157 
13. Acharya Sodhala's Sodhala Nighantu Text With English- Hindi Commentaries; Prof. (Dr.) Gyanendra Panday, Chowkhamba Krishnadas Academy Varansi , Edition First 2009 PgNo 161-178

14. Prof. (Dr.) Gyanendra Panday, Acharya Sodhala's Sodhala Nighantu Text With English- Hindi Commentaries; Chowkhamba Krishnadas Academy Varansi, Edition First 2009 PgNo 179-196

15. Prof. (Dr.) Gyanendra Panday,Acharya Sodhala's Sodhala Nighantu Text With English- Hindi Commentaries; Chowkhamba Krishnadas Academy Varansi, Edition First 2009 PgNo 197-490

\section{Source of Support: Nil \\ Conflict of Interest: None Declared}

How to cite this URL: Nishi Choudhary et al: An Introduction About Sodhala Nighantu. International Ayurvedic Medical Journal \{online\} 2021 \{cited February, 2021\} Available from: http://www.iamj.in/posts/images/upload/415_422.pdf 